\title{
Avaliação do Sistema de Monitoramento Computadorizado de Digestão In Vitro. 1. Testes Preliminares ${ }^{1}$
}

\author{
Fábio Prudêncio de Campos ${ }^{2}$, Dante Pazzanese Duarte Lanna ${ }^{3}$, Máx Lázaro Vieira Bose $^{4}$, Celso Boin $^{5}$
}

\begin{abstract}
RESUMO - O objetivo deste trabalho foi testar o sistema de monitoramento computadorizado da produção de gás in vitro. Essa técnica facilita estudos da degradação das frações solúveis e insolúveis das forragens, quantificadas pela produção de gás $\left(\mathrm{CO}_{2}\right.$ e $\left.\mathrm{CH}_{4}\right)$ oriunda do metabolismo microbiano e medida por sensor de pressão. Diversas quantidades de amostras (50 a $110 \mathrm{mg}$ de feno de alfafa) e outros alimentos foram testadas. Também, a influência da quantidade de líquido ruminal (2,0 ou 3,0 mL), com ou sem barras magnéticas agitadoras nos frascos incubatórios, na digestibilidade de $100 \mathrm{mg}$ de feno de alfafa, foi estudada. A quantidade de $100 \mathrm{mg}$ de amostra, 2,0 mL de líquido ruminal e sem barra magnética agitadora, proporcionou os menores coeficientes de variação na produção de gás. Em conclusão, 2,0 mL de líquido ruminal, sem barra e com $100 \mathrm{mg}$ de amostra, apresentaram maior precisão na curva de digestão.
\end{abstract}

Palavras-chave: digestão in vitro, forragem, produção de gás

\section{Evaluation of a Computerized Monitoring on In Vitro Digestion System. 1. Preliminary Tests}

\begin{abstract}
The objective of this work was to test the computerized monitoring of the in vitro gas production system. This technique made easy the degradation studies of soluble and insoluble fractions of forages, determined by the gas production $\left(\mathrm{CO}_{2} \mathrm{e} \mathrm{CH}_{4}\right)$ from microbial metabolism and measured by a pressure sensor. Several amounts of samples (50 to $110 \mathrm{mg}$ of alfalfa hay) and other feedstuffs were tested. Also, the influence of the amount of ruminal liquid (2.0 or $3.0 \mathrm{~mL}$ ), with or without stirs in incubator flasks, on the digestibility of $100 \mathrm{mg}$ of alfalfa hay, was studied. The amount of $100 \mathrm{mg}$ of sample, $2.0 \mathrm{~mL}$ of ruminal liquid and without agitating magnetic bar, provided lower coefficients of variance on the gas production. In conclusion, $2.0 \mathrm{~mL}$ of ruminal liquid, without stirrer with $100 \mathrm{mg}$ of sample, presented a greater precision of the digestion curve.
\end{abstract}

Key Words: in vitro digestion, forage, gas production

\section{Introdução}

A eficiência de produção bovina em sistemas intensivos é, em grande parte, atribuída ao balanceamento adequado da ração. Formulações eficientes e econômicas dependem do valor nutritivo dos ingredientes. Portanto, novas metodologias de avaliação de alimentos têm sido um processo constante. Entretanto, o método de digestibilidade in vitro (GOERING et al., 1970; TILLEY E TERRY, 1963) é ainda o mais usual e apresenta grandes problemas (PELL e SCHOFIELD 1993), como: 1) a análise de fibra destrói a amostra e são requeridas novas amostras para novas análises; 2) o início do estádio da digestão é dificultado pela maior quantidade de amostra; 3) os componentes solúveis das forragens não podem ser determinados; 4) necessitam de volume maior de amostras para ser possível realizar todas as análises. Uma alternativa é a medida do desaparecimento ponderal (MENKE e STEINGASS, 1988; TAYA et al. 1980) da fibra pela produção de gás, em decorrência da atividade microbiana no rúmen. WILKINS (1974) já havia descrito um sistema automático, em que a produção de gás era registrada em mapa gráfico. Evoluindo os estudos, TAYA et. al. (1980) relataram, em seus trabalhos, que a produção de gás tem efeito linear com a digestão de celulose incubada com Ruminococus albus. MENKE e STEINGASS (1988) observaram que a produção de gás no processo fermentativo, a qual tem sido medida manometricamente, proporciona energia para os ruminantes. Outra alternativa encontrada foi a medição da pressão do gás em sistema fechado ou volumetricamente (MENKE e STEINGASS, 1988). Trabalhando nesta linha, PELL e SCHOFIELD (1993) aperfeiçoaram a técnica de digestão in vitro por

\footnotetext{
1 Projeto financiado pela FAPESP e pelo CNPq.

2 Zoot./MS - Dados parciais de Dissertação de Mestrado - ESALQ/USP; Doutorando na FCAVJ/UNESP. E.mail: fpcampos 99@yahoo.com

3 Professor no Departamento de Zootecnia - ESALQ/USP. E.mail: dplanna@esalq.usp.br

4 Professor aposentado no Departamento de Zootecnia - ESALQ/USP.

5 Professor aposentado no Departamento de Zootecnia - ESALQ/USP.
} 


\section{6}

intermédio da medição de gás por sensores de pressão em sistema fechado e computadorizado. Constataram o desaparecimento de fibra pelo gás produzido no metabolismo microbiano, por meio de sensores de pressão conectados aos frascos incubatórios e ao computador com software apropriado, que monitora as leituras efetuadas ao longo do tempo de incubação.

O funcionamento do sensor de pressão baseia-se na mudança da resistência e deformação do filme semicondutor de silicone. Um circuito de ponte de Wheatstone é usado para detectar essa mudança de resistência, e o sinal do sensor aparece como uma mudança de voltagem, a qual é correlacionada linearmente com a mudança de pressão e, assim, pode ser convertida em volume de gás, quando injetado em recipiente fechado, sistema de volume fixo (SCHOFIELD et al., 1994).

Outras técnicas de digestibilidade da matéria seca, pela produção de gás, pelos sistemas manométricos e ou por transdutor de pressão, vêm sendo realizadas no Brasil, com o objetivo de avaliar a forrageiras tropicais. Essas técnicas possuem os mesmos princípios que as demais, só diferenciam no protocolo de análise. MALAFAIA et al. (1998), avaliando a cinética da degradação da matéria seca de alguns volumosos produzidos no Brasil, como capim-tifton, capim-elefante, algumas braquiárias, soja perene e silagem de milho, pelos diferentes métodos in situ, in vitro e pela produção de gás - sistema manométrico, concluíram que a determinação manométrica e volumétrica da produção de gás apresentou menores variações nas mensurações, sendo, portanto, recomendada para estimar as taxas de degradação dos alimentos utilizados na alimentação de ruminantes.

Diversas variáveis, incluindo tamanho da amostra, quantidade de inóculo, tamanho do recipiente e tipo de sensor de pressão, têm sido investigadas para a determinação da faixa de produção de gás, que pode ser medida adequadamente com precisão dos resultados (PELL e SCHOFIELD 1993).

Os objetivos do presente trabalho foram avaliar o sistema de monitoramento computadorizado da produção de gás na digestão de alimentos in vitro e constatar a eficiência desse sistema.

\section{Material e Métodos}

Os componentes do sistema de monitoramento computadorizado, contínuo, da produção de gases da digestão in vitro e do esquema do equipamento (Figura 1) descrito por PELL e SCHOFIELD (1993) estão relacionados a seguir. Frascos de incubação: vidro de $50 \mathrm{~mL}$, tampa de borracha e lacre de alumínio; estufa: caixa de madeira compensada, de espessura de

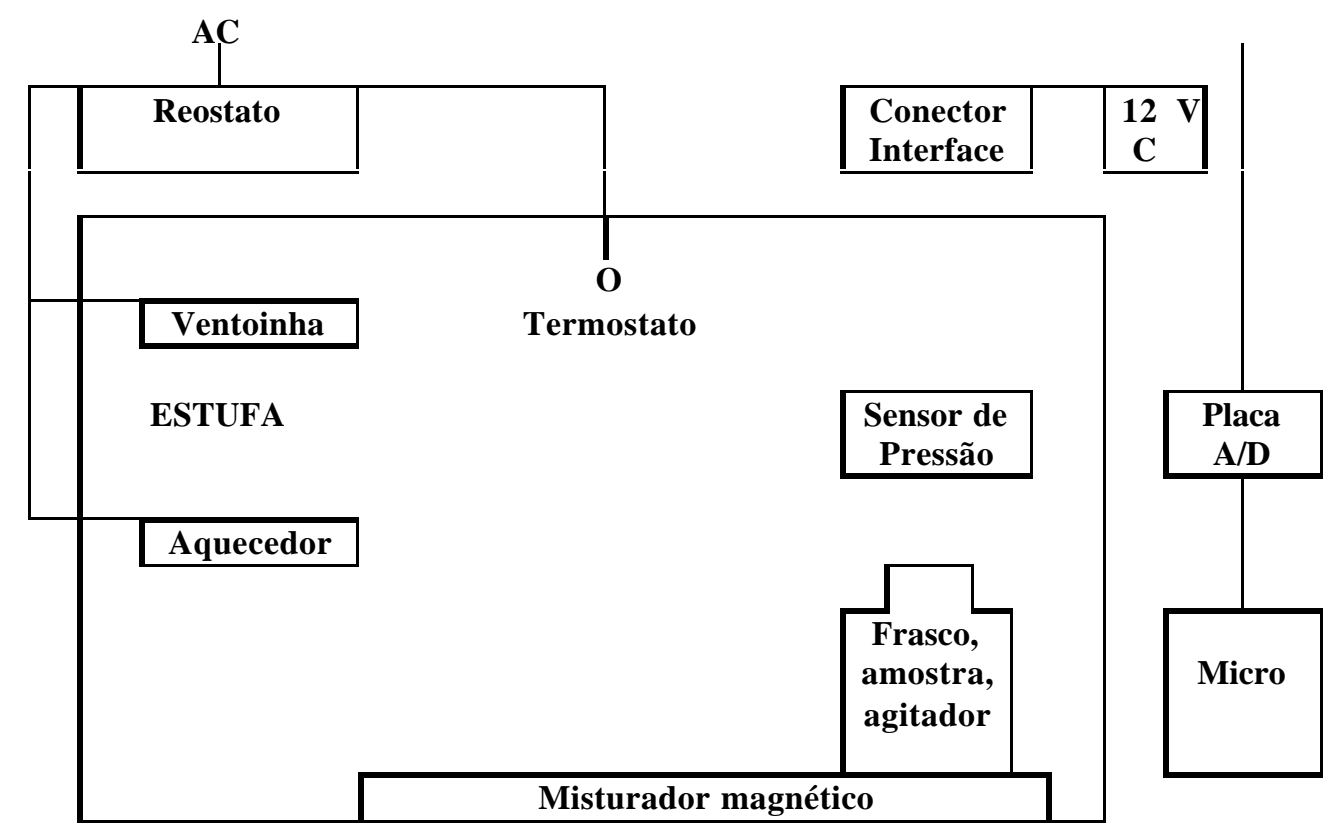

Figura 1 - Esquema do sistema de digestão in vitro computadorizada. A abreviação AC indica fonte de corrente elétrica alternada; C, conversor de energia elétrica para 12 volts; e A/D, conversor analógico/digital, conforme PELL e SCHOFIELD - 1993 (adaptado).

Figure 1 - Scheme of the in vitro digestion computerized system. The abbreviation AC stands for source of electrical alternate current, $C$, the electrical energy converter for 12 volts and A/D, analogical/digital converter, according to PELL and SCHOFIELD - 1993 (adapted). 
$20 \mathrm{~mm}$, com as seguintes dimensões $70 \times 70 \times 60 \mathrm{~cm}$, com suporte para 16 frascos de vidro de $50 \mathrm{~mL}$, três lâmpadas de $90 \mathrm{~W}$, termostato para controle da temperatura interna da estufa $\left(39^{\circ} \mathrm{C}\right)$ e ventoinha de circulação do ar; misturadores: constituídos de quatro motores de $48 \mathrm{rpm}$, instalados abaixo do suporte de cada quatro frascos de incubação, acoplado a um suporte metálico com duas barras magnéticas de aproximadamente $1,0 \times 1,0 \mathrm{~cm}$, que movimentam as barras magnéticas agitadoras no interior dos frascos incubatórios; sensores de pressão: em número de 16 peças, apresentam circuitos fechados, possuem diafragmas sensíveis a uma faixa de pressão de 0 a 103,4 Kpa ( 0 a 15 psi) e requerem fonte de corrente elétrica contínua de $12 \mathrm{~V}$; placa interface A/D: componente que apresenta amplificador programável para os sensores e para leitura dos 16 canais; computador: IBM 80386, 40 Mhz, com quatro Megabytes de memória (configuração mínima), estabilizador de energia elétrica e um equipamento que evita a interrupção da energia elétrica ("Nobreak's); e programa: "Signal View - 1.3 aquisição e monitoramento de dados", adquirido da "Real TimeDevices", sendo suporte doamplificador programável característico da placa interface A/D. A versão utilizada do software é a de 16 canais para Windows 3.0 ou 3.1.

As soluções ou misturas utilizadas seguiram metodologias descritas por MENKE e STEINGASS (1988) e GOERING e VAN SOEST (1970): mistura tampão: 4,0 g de $\left(\mathrm{NH}_{4}\right) \mathrm{HCO}_{3}, 35,0 \mathrm{~g}$ de $\mathrm{NaHCO}_{3}$, por litro de água destilada; mistura de macro minerais: 5,7 $\mathrm{g} \mathrm{Na}_{2} \mathrm{HPO}_{4}$ anidro, 6,2 $\mathrm{g} \mathrm{KH}_{2} \mathrm{KO}_{4}$ anidro, 0,6 $\mathrm{g}$ $\mathrm{MgSO}_{4}+7 \mathrm{H}_{2} \mathrm{O}$ completados em 1 litro de água destilada. É recomendado que se diluam primeiramente os fosfatos em 800 a $900 \mathrm{~mL}$ de água, a fim de evitar precipitado insolúvel. Mistura de micro minerais: $13,2 \mathrm{~g}$ $\mathrm{CaCl}_{2}+2 \mathrm{H}_{2} \mathrm{O}, 10,0 \mathrm{~g} \mathrm{MnCl}_{2}+4 \mathrm{H}_{2} \mathrm{O}, 1,0 \mathrm{~g} \mathrm{CoCl}_{2}+$ $6 \mathrm{H}_{2} \mathrm{O}, 8,0 \mathrm{~g} \mathrm{FeCl}_{3}+6 \mathrm{H}_{2} \mathrm{O}$ completados em $100 \mathrm{~mL}$ de água destilada. Para cada teste da digestibilidade, foi preparada uma solução nutritiva com $0,4 \mathrm{~g}$ de caseína como fonte de proteína, $80 \mathrm{~mL}$ de $\mathrm{H}_{2} \mathrm{O}$ destilada, $40 \mathrm{~mL}$ de solução tampão, $40 \mathrm{~mL}$ de solução de macro mineral, $20 \mu \mathrm{L}$ de solução de micro mineral, $200 \mu \mathrm{L}$ de resazurina a $0,1 \%$ e solução digestora de $3,0 \mathrm{~mL}$ de $\mathrm{HCl} 1 \mathrm{~N}$. Estes componentes foram colocados em balão volumétrico de fundo redondo, boca fechada com papel alumínio e aquecido em bico de Bunsen, evitando atingir a fervura. Promoveu-se resfriamento em recipiente com gelo até temperatura de $39^{\circ} \mathrm{C}$, adicionando-se $100 \mathrm{mg}$ de $\mathrm{HCl}$ cisteína, injetando-se, ao mesmo tempo, fluxo constante de $\mathrm{CO}_{2}$ dentro do balão, a fim de se evitar entrada de oxigênio. Mediu-se o pH, que deveria estar em torno de 6,8 a 6,9 e, se necessário, corrigir-se-ia com adição de $\mathrm{NaOH}$ ou $\mathrm{HCl}$. Após todos esses procedimentos, obteve-se a denominada mistura média. Os frascos incubatórios, nesta fase, já deveriam estar com $100 \mathrm{mg}$ de amostra de alimento previamente pesada, seca a $60^{\circ} \mathrm{C}$ e moída a $1 \mathrm{~mm}$, mais $0,5 \mathrm{~mL}$ de água destilada, para evitar perda de amostra por dispersão, ao injetar $\mathrm{CO}_{2}$. O branco foi um frasco incubatório com mistura média e sem amostra de alimentos. Em seguida, foram adicionados $8 \mathrm{~mL}$ de mistura média dentro de cada frasco com fluxo constante de $\mathrm{CO}_{2}$, fechou-se com tampa de borracha lubrificada com vaselina em pasta, lacrou-se a tampa com anel de alumínio e vazou-se rapidamente cada frasco, a fim de deixar padronizada a pressão interna dos mesmos. Após, foram colocados na estufa e aguardou-se equilíbrio da temperatura. Logo em seguida, iniciou-se o processo de calibração, que consistiu em vazar os frascos, rodando o programa com $0 \mathrm{~mL}$ de $\mathrm{CO}_{2}$, após 20 minutos adição de $10 \mathrm{~mL} \mathrm{de} \mathrm{CO}_{2}$. O objetivo desta calibração foi obter projeção linear das leituras de 0 e $10 \mathrm{~mL}$ de $\mathrm{CO}_{2}$ e, também, o coeficiente "b" da equação linear ( $Y=a \pm b x)$, que possibilitaria a correção e transformação dos dados lidos, em volume de gás produzido e em função da pressão barométrica do dia. Foi utilizado líquido ruminal de bovino fistulado no rúmen, filtrado em dois "panos de queijo" em quatro camadas e colocado em garrafa térmica. Em seguida, foi filtrado novamente em pano e em camada de $2 \mathrm{~cm}$ lã de vidro, com a injeção contínua de $\mathrm{CO}_{2}$, transferido para frasco de $200 \mathrm{~mL}$, que foi lacrado, e colocado em estufa para manter temperatura. Após a calibração, foi injetado o líquido ruminal em cada frasco, tomando-se sempre o cuidado de evitar a penetração de ar. Testouse a influência da adição ou não de barras magnéticas agitadoras, no interior dos frascos incubatórios, na digestão de feno de alfafa incubada em triplicatas de amostras com 2 ou $3 \mathrm{~mL}$ líquido ruminal. Essas barras tinham a função de movimentar as amostras dos alimentos no meio líquido, a fim de proporcionar maior contato entre microrganismos e partículas de alimentos. Testouse, ainda, a influência de diferentes quantidades de fenos de alfafa $(50,60,70,80,90,100$ e $110 \mathrm{mg})$ sobre a produção de gás e efeito sobre $\mathrm{o} \mathrm{pH}$ ao final da digestão de 48 horas. O delineamento estatístico para estas avaliações foi em blocos casualizados, com sete níveis (quantidades de amostras), três repetições analisadas por regressão polinomial. Para os demais volumosos, como a silagem de milho com $41,9 \%$ MS e $35 \%$ de 
grãos, a silagem de milho com 37\% MS inoculada com lactobacilos e as folhas de capim-elefante colhido com 45 , $55,65,75,85$ e 95 dias, utilizaram-se $100 \mathrm{mg}$ de amostra, a fim de determinar o efeito sobre os coeficientes de variação e a repetibilidade das mensurações, como volume total de gás e pH após 48 horas de incubação.

\section{Resultados e Discussão}

As diferenças nos resultados de produção de gás dos volumosos analisados podem ser causadas pelas diferentes amostragens dos 2,0 $\mathrm{mL}$ de líquido ruminal do reservatório. A homogeneização do líquido ruminal do reservatório antes da coleta não garante a seleção das diferentes quantidades e espécies de microorganismos do inóculo existente. SCHOFIELD e PELL (1995), ao usarem diferentes inóculos de líquido ruminal, encontraram diferenças significativas, as quais foram atribuídas a diferentes quantidades e espécies de microrganismos no inóculo. Nesta mesma linha de pesquisa, NELSON et al. (1973) encontraram diferença significativa $(\mathrm{P}<0,01)$ na digestibilidade da matéria seca in vitro de alimentos com inóculos de vacas consumindo alimentos diferentes das analisadas.

Foram feitos testes com e sem a adição de barras magnéticas no interior dos frascos incubatórios associados com 2,0 ou 3,0 mL de líquido ruminal e $100 \mathrm{mg}$ de amostra (Tabela 1). Foi obtido coeficiente de variação na produção total de gases de 5,2\% com a barra magnética e 1,3\% sem a barra, com 2,0 mL de líquido ruminal, contra 2,1 e $4,2 \%$, respectivamente, obtidos por PELL e SCHOFIELD (1993). Provavelmente, a diferença de imediato esteja em função do movimento circular e oscilatório apresentado pelas barras magnéticas agitadoras. Entretanto, pode ter ocorrido interação

Tabela 1 - Valores médios da produção total de gás, em função dos volumes de líquido ruminal (2 ou $3 \mathrm{~mL}$ ), por frasco incubatório com (C.B.) ou sem (S.B.) barra magnética agitadora, triplicatas de $100 \mathrm{mg}$ de amostra

Table 1 - Mean values of total gas production in function of volumes of the ruminal liquid ( 2 or $3 \mathrm{~mL}$ ) perincubatory frask with (W.B.) or without (Wo.B.) agitating magnetic bar, triplicate of $100 \mathrm{mg}$ sample

\begin{tabular}{lccr}
\hline $\begin{array}{l}\text { Líquido ruminal } \\
\text { Ruminal liquid }\end{array}$ & $\begin{array}{c}\text { Volume de } \\
\text { gás (mL) } \\
\text { Gas volume }\end{array}$ & $\begin{array}{c}\text { Desvio-padrão } \\
\text { Standard deviation }\end{array}$ & CV (\%) \\
\hline 3 mL - C.B. (W.B.) & 22,4 & 4,98 & 22,3 \\
3 mL - S.B. (Wo.B.) & 16,8 & 1,98 & 11,8 \\
2 mL - C.B. (W.B.) & 20,2 & 1,05 & 5,2 \\
2 mL - S.B. (Wo.B.) & 18,7 & 0,24 & 1,3 \\
\hline
\end{tabular}

entre o tipo de alimento e o tipo de líquido ruminal com o mecanismo oscilatório da barra magnética nos frascos incubatórios. Quando se utilizaram $3 \mathrm{~mL}$ de líquido ruminal associados ou não com a utilização de barra magnética agitadora, obtiveram-se maiores coeficientes de variação, 22,3\% para produção total de gases com barra e $11,8 \%$ sem barra magnética. Essa variação pode ter ocorrido devido ao efeito negativo da aderência de partículas de amostras na parede dos frascos, em função do movimento circular e oscilatório da barra magnética agitadora.

Dentro de cada rodada testada, foi avaliada a repetibilidade dos dados de volume total de gás ao final da digestão, a quantidade e tipo de amostra e o pH final de cada alimento incubado, por meio de médias, análises de regressão polinomial e seus respectivos coeficiente de variação, para cada tratamento (Tabela 2). Utilizouse o período de digestão de 48 horas pela produção gás, devido à baixa disponibilidade substrato na fermentação e/ou morte dos microrganismo, com o abaixamento do pH. Pode-se constatar, por intermédio dos dados obtidos, que, quanto menor a quantidade de amostra de alimentos, maior será o coeficiente de variação entre as repetições. Foi observado, pela análise de regressão polinomial, efeito quadrático e significativo $(\mathrm{P}<0,05)$ da produção máxima de gás, pelas quantidades de amostras incubadas. A quantidade ideal de amostra ficou na faixa de 80 a $90 \mathrm{mg}$ (Figura 2), porém a quantidade de $100 \mathrm{mg}$ apresentou menores variações nas repetições (CV 0,5\%), indicando que há proporcionalidade entre a relação líquido:amostra (1:10) proposta por GOERING e VAN SOEST (1970); também, quanto maior a quantidade de amostra, menor o $\mathrm{pH}$ do líquido residual. Pela análise de regressão polinomial, foi observado efeito linear e significativo $(\mathrm{P}<0,05)$, quando se avaliaram a influência de quantidade de amostra e o $\mathrm{pH}$ ao final da digestão de 48 horas de incubação de feno de alfafa (Figura 3). Este é um fator essencial para o crescimento microbiano, o que, provavelmente, indique a necessidade de certa cautela, quando se utilizam alimentos concentrados.

A técnica manométrica estudada por MALAFAIA et al. (1998), a qual utilizou frascos de $100 \mathrm{~mL}$, contendo $75 \mathrm{~mL}$ de meio de cultura, 5 $\mathrm{mL}$ de inóculo ruminal e $360 \mathrm{mg}$ de amostra de alimento e mensurações da pressão e volumes nos tempos $0,5,1,2,3,4,6,9,12,18,24,30,36,48$, 72 e 96 horas após incubação, por meio de manômetros e seringas graduadas, distingue-se 


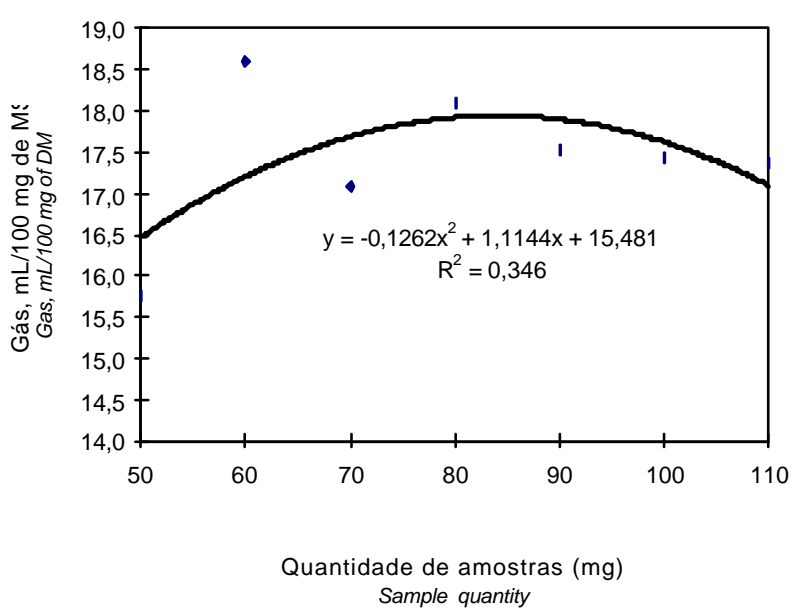

Figura 2 - Análises de regressão polinomial da produção de gás $(\mathrm{mL})$ nas diferentes proporções de amostras de feno de alfafa (FA), em triplicatas, após 48 horas de incubação.

Figure 2 - Polynomialregressionanalysis of gas production $(m L)$ in different proportions of alfalfa hay samples (HA), triplicate samples, after $48 \mathrm{~h}$ of incubation.

da técnica in vitro/gás computadorizada, por não realizarem leituras contínuas e automatizadas durante o período de incubação adotado. As leituras são realizadas nos tempos programados, em milivolts, e transformadas em volume de gás pelo uso das calibrações iniciais e uso dos coeficientes de regressão linear para a correção da produção de gás, não diferenciando das correções obtidas nos métodos que utilizam dos sistemas manométricos e volumétrico (THEODOROU et al., 1994; MALAFAIA et al., 1998), que corrigem a produção de gás pelos coeficientes de regressão linear entre volume e pressão do gás.

As diferenças nas proporções de amostra e líquido nos frascos incubatórios $(1: 4,5)$ utilizados por MALAFAIA et al. (1998) estão abaixo do proposto por GOERING e VAN SOEST (1970). Na técnica in vitro/gás computadorizada, o aumento na quantidade de amostra ou líquido excederia as proporções definidas como ideais e, conseqüentemente, poderiam causar variações no $\mathrm{pH}$ e nas repetibilidades das leituras avaliadas pelos coeficientes de variação (Tabelas 1 e 2 e Figura 3).

Foi observado também que, na avaliação da repetibilidade dos resultados da produção cumulativa de gás, pelo coeficiente de variação, da silagem de milho e das folhas do capim-elefante nos diversos tempos de corte, o sistema in vitro/gás computadorizado se apresentou como alternativa eficiente para as futuras avaliações das taxas de degradações desses alimentos. MALAFAIA et al. (1998)

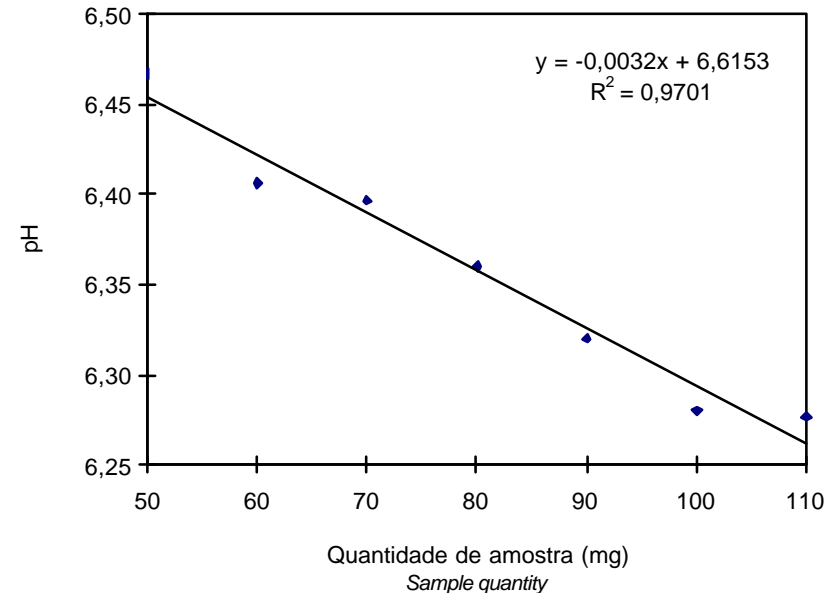

Figura 3 - Análise de regressão polinomial da influência da quantidade de amostra $(\mathrm{mg})$ no $\mathrm{pH}$ do resíduo da digestão in vitro, do feno de alfafa, em triplicatas, após 48 horas de incubação.

Figure 3 - Polynomial regression analysis of the influence of the sample amount $(\mathrm{mg})$ on the $\mathrm{pH}$ of the residue of digestion in vitro, of the alfalfa hay, triplicate samples, after $48 \mathrm{~h}$ of incubation.

encontraram coeficiente de variação das análises de digestibilidade da matéria seca do capim-elefante com 60 dias e silagem de milho, pela produção de gás, próximo ao encontrado neste trabalho $\cong 2,0$ e 2,5 contra $\cong 2,0$ e $3,0 \%$, respectivamente).

Neste experimento, detectou-se que a calibração e o branco são pontos essenciais no sistema, pois todos os demais dados são condicionados a eles. Entretanto, a calibração influi na resposta do branco, sendo, portanto, o ponto chave para se obterem resultados precisos. Se o gás usado na calibração, ou medido pelo sensor em uso, for solúvel na solução média, parte deste será dissolvido e acarretará aumento na pressão. Essa medida é governada pela Lei Henry's, em que independente do estado da fração molar do gás dissolvido este será proporcional à pressão parcial em equilíbrio na fase líquida, sendo, portanto, necessário à correção dos dados, com a seguinte fórmula adaptada de PELL e SCHOFIELD (1995): $\mathrm{Fc}=\{\mathrm{Fb} *(\mathrm{Pat} / \mathrm{P}) /[(\mathrm{Vf}-0,371 * \mathrm{Vs}) /(\mathrm{Vf}-0,371 * \mathrm{Vfi})]\}$ em que $\mathrm{Fc}$, fator de correção; $\mathrm{Fb}$, fator b da equação linear obtida entre o volume zero e $10 \mathrm{~mL}$ de $\mathrm{CO}_{2}$ no processo de calibração inicial; Pat, pressão barométrica do dia; $\mathrm{P}$, pressão atmosférica a nível do mar $(760 \mathrm{mmHg})$; Vf, volume do frasco; 0,371, valor obtido em função Lei de Henry's; Vs, volume solução; e Vfi, volume da solução + inóculo. Portanto, dos gases provenientes da fermentação anaeróbia da celulose, apenas o $\mathrm{CO}_{2}$ tem significante solubilidade em meio líquido à pressão atmosférica. 
Tabela 2 - Valores médios e coeficiente de variação da produção de gás e pH nas diferentes proporções de amostras de feno de alfafa (FA), silagem de milho inoculada (SMI), silagem de milho (SM) e folhas de capim-elefante (FCE), triplicatas de $100 \mathrm{mg}$ de amostras

Table 2 - Mean values and coefficient of variantion of gas production and $\mathrm{pH}$ in the different proportions of alfalfa hay samples (HA), innoculated corn silage (CSI), corn silage (CS) and elephant grass leaves (LEG), triplicate of $100 \mathrm{mg}$ sample

\begin{tabular}{|c|c|c|c|c|c|}
\hline $\begin{array}{l}\text { Forragem } \\
\text { Forage }\end{array}$ & $\begin{array}{c}\text { Quantidade de amostra (mg) } \\
\text { Amount of samples }\end{array}$ & $\begin{array}{c}\text { Volume de gás - Média }(\mathrm{mL}) \\
\text { Gas volume - Mean }\end{array}$ & $\mathrm{CV}(\%)$ & $\begin{array}{l}\mathrm{pH} \text { médio } \\
\text { Average } \mathrm{pH}\end{array}$ & $\mathrm{CV}(\%)$ \\
\hline & 50 & 15,8 & 4,1 & 6,5 & 0,8 \\
\hline & 60 & 18,8 & 2,7 & 6,4 & 0,1 \\
\hline $\mathrm{FA}^{1}$ & 70 & 17,1 & 10,2 & 6,4 & 0,9 \\
\hline \multirow[t]{4}{*}{$H A$} & 80 & 18,1 & 3,4 & 6,4 & 0,3 \\
\hline & 90 & 17,5 & 1,6 & 6,3 & 0,3 \\
\hline & 100 & 17,4 & 0,5 & 6,3 & 0,0 \\
\hline & 110 & 17,4 & 2,0 & 6,3 & 0,4 \\
\hline SMI & 100 & 17,5 & 0,6 & 6,3 & 0,4 \\
\hline CSI & & & & & \\
\hline SM & 100 & 20,4 & 3,0 & 6,2 & 1,1 \\
\hline \multirow{2}{*}{\multicolumn{5}{|c|}{$\begin{array}{l}\text { CS } \\
\text { FCE }\end{array}$}} & \\
\hline & & & & & \\
\hline \multicolumn{6}{|l|}{$L E G$} \\
\hline 45 dias & 100 & - & 2,78 & 6,33 & 3,3 \\
\hline 55 dias & 100 & - & 1,85 & 6,56 & 3,2 \\
\hline 65 dias & 100 & - & 2,04 & 6,53 & 0,3 \\
\hline 75 dias & 100 & - & 2,59 & 6,52 & 1,1 \\
\hline 85 dias & 100 & - & 3,21 & 6,45 & 0,3 \\
\hline 95 dias & 100 & - & 2,32 & 6,46 & 0,4 \\
\hline
\end{tabular}

${ }^{1}$ Avaliado por análises de regressão polinomial.

SMI - Silagem de milho com $37 \%$ MS e inoculada com Lactobacilos.

SM - Silagem de milho com $41,9 \%$ MS e $35,0 \%$ de grãos.

1 Evaluate for analysis of polynomial regression.

CSI - Corn silage with $37 \%$ DM and inoculated with Lactobacillus.

CS - Corn silage with $41,9 \%$ DM and $35 \%$ of grain.

\section{Conclusões}

Concluiu-se que 2,0 mL de líquido ruminal, sem barra, e 90 a $110 \mathrm{mg}$ de amostra mostraram ser a faixa ideal para a manutenção do $\mathrm{pH}$ e, conseqüentemente, maior precisão na curva de digestão.

A calibração e o branco são pontos essenciais no sistema, pois todos os demais dados são condicionados a eles. Entretanto, a calibração influi na resposta do branco, sendo, portanto, o ponto chave para se obterem resultados precisos.

O sistema in vitro/gás computadorizado apresentou-se como alternativa eficiente para as futuras avaliações das taxas de degradações de volumosos, pelos menores coeficiente de variação apresentados.

\section{Referências Bibliográficas}

GOERING, K.H., VAN SOEST, P.J. 1970. Forage fiber analysis (apparatus, reagents, procedures, and some application). Agricultural Handbook, Agricultural Research Council. Washington, D.C.: US. Department of Agriculture. 379p. MALAFAIA, P.A.M., VALADARES FILHO, S.C., VIEIRA, R.A.M. et al. 1998. Cinética ruminal de alguns alimentos investigada por técnicas gravimétricas e metabólicas. $R$. Bras. Zootec., 27(2):370-380.
MENKE, K.H., STEINGASS, H. 1988. Estimation of the energetic feed value obtained from chemical analysis and in vitro gas production using rumen fluid. Anim. Res. Dev., 28(1):7-55.

PELL, A.N., SCHOFIELD, P. 1993. Computerized monitoring of gas production to measure forage digestion in vitro. J. Dairy Sci., 76(4):1063-1073.

SCHOFIELD, P., PITT, R.E., PELL, A.N. 1994. Kinetics of fiber digestion from in vitro gas production. J. Anim. Sci., 72(11):2980-2991.

TAYA, M., OHMIYA, K., KOBAYASHI, T. et al. 1980. Monitoring and control of a cellulolytic anaerobe culture by using gas envolved as an indicator. J. Ferment. Technol., 5:463-470.

THEODOROU, M.K., WILLIAMS, B.A., DHANOA, M.S. et al. 1994. A simple gas production method using a pressure transducer to determine the fermentation kinetics of ruminant feed. Anim. Feed. Sci. Technol., 48(1):185-197.

TILLEY J.M.A., TERRY R.A. 1963. A two-stage technique for the in vitro digestion of forrage crops. J. Br. Grass. Soc., 18:104-111.

WILKINS, J.R. 1974. Pressure transducer method for measuring gas production by microorganisms. Appl. Microbiol., 27:135-141.

Recebido em $31 / 10 / 97$

Aceito em 27/09/99 